\title{
Coronavirus Outbreak and the Mathematical Growth Map of COVID-19
}

\author{
Md. Kamrujjaman ${ }^{1,2^{*}}$, Md. Shahriar Mahmud ${ }^{1}$ and Md. Shafiqul Islam ${ }^{3}$ \\ ${ }^{1}$ Department of Mathematics, University of Dhaka, Dhaka 1000, Bangladesh. \\ ${ }^{2}$ Department of Mathematics and Statistics, University of Calgary, Calgary, AB, Canada. \\ ${ }^{3}$ School of Mathematical and Computational Sciences, University of Prince Edward Island, \\ Charlottetown, PE, Canada.
}

Authors' contributions

This work was carried out in collaboration among all authors. Author MK designed the study, performed the statistical analysis, wrote the protocol, and wrote the first draft of the manuscript. Authors MSM and MSI managed the analyses of the study, the literature searches and revised the final draft. All authors read and approved the final manuscript.

Article Information

DOI: $10.9734 / A R R B / 2020 / v 35 i 130182$

Editor(s):

(1) Paola Angelini, University of Perugia, Italy.

Reviewers:

(1) Wachira Charles, Masinde Muliro University of Science and Technology, Kenya.

(2) Segun. I. Oke, University of Pretoria, South Africa.

Complete Peer review History: http://www.sdiarticle4.com/review-history/55773

Original Research Article

Received: 16 March 2020

Accepted: 24 March 2020

Published: 26 March 2020

\section{ABSTRACT}

In the last two decades the world had faced three respiratory syndrome outbreaks incurred by Coronavirus. Though the wild animals are the primary carriers of the virus, the human population managed to survive sacrificing more than 1,600 lives from 2002 to 2012 . But the current virus outbreak has already taken more than 2,462 lives since 22 February 2020. In the first few days, when the cases were being introduced under light, there were no treatment for the infection and the unleashed spread demands to be analyzed to see the pattern of the outbreak. This manuscript aims to look into the growth map of the COVID-19 outbreak under mathematical growth functions and tries to understand which growth pattern assembles the scenario for the cases.

Keywords: Population dynamics; growth rate; real life data.

2010 Mathematics Subject Classification: 92D30, 92D25, 34C11.

\footnotetext{
*Corresponding author: E-mail: kamrujjaman@du.ac.bd;
} 


\section{INTRODUCTION}

Coronaviruses are the family of viruses that can cause illnesses such as the common cold, severe acute respiratory syndrome (SARS) and Middle East respiratory syndrome (MERS) which may lead to death. In December 2019, a new virus called the severe acute respiratory syndrome coronavirus 2 (SARS-CoV-2) caused a disease outbreak in China. The disease is called coronavirus disease 2019 (COVID-19). WHO (World Health Organization) says, Coronaviruses are zoonotic, which means they are transmitted between animals and people. Investigations found that SARS-CoV was transmitted from civet cats to humans, MERS-CoV from dromedary camels to humans and it is believed that the COVID-19 from wild bat or snake to humans $[1,2,3]$. Several known coronaviruses are circulating in animals that have not yet infected humans [4].

The SARS epidemic, which began in China in 2002-2003, killed 774 people worldwide with a huge 8,000 number of infection cases $[5,6]$. There had been only two confirmed deaths from the new coronavirus outside mainland China: one in Hong Kong and one in the Philippines.

Since September 2012, WHO has been notified of 2, 494 laboratory-confirmed cases of infection with MERS-CoV, 858 MERS-CoV associated deaths and 27 countries [5] have reported cases of MERS-CoV. After analysis and comparing the genetic sequences of COVID-19 with other known coronavirus, scientists have suggested and predicted that the new virus is most closely related to two bat SARS-like coronavirus samples from China and the virus has been transformed from snake to human as snake often hunts for bats [7].

The recent appearance of the 2019 coronavirus was first observed in early December 2019 in Wuhan, a major city in central China. All the latest updates to date February 21, 2020, coronavirus death toll on mainland China reaches more than 2,462 , while the number of confirmed cases is 78,773 according to the ALA (American Library Association) certified worldometer [8], where $99.19 \%$ of them are from China, the heart of the outbreak. WHO has already announced Global Health Emergency in this critical condition created by COVID-19 Coronavirus [5].

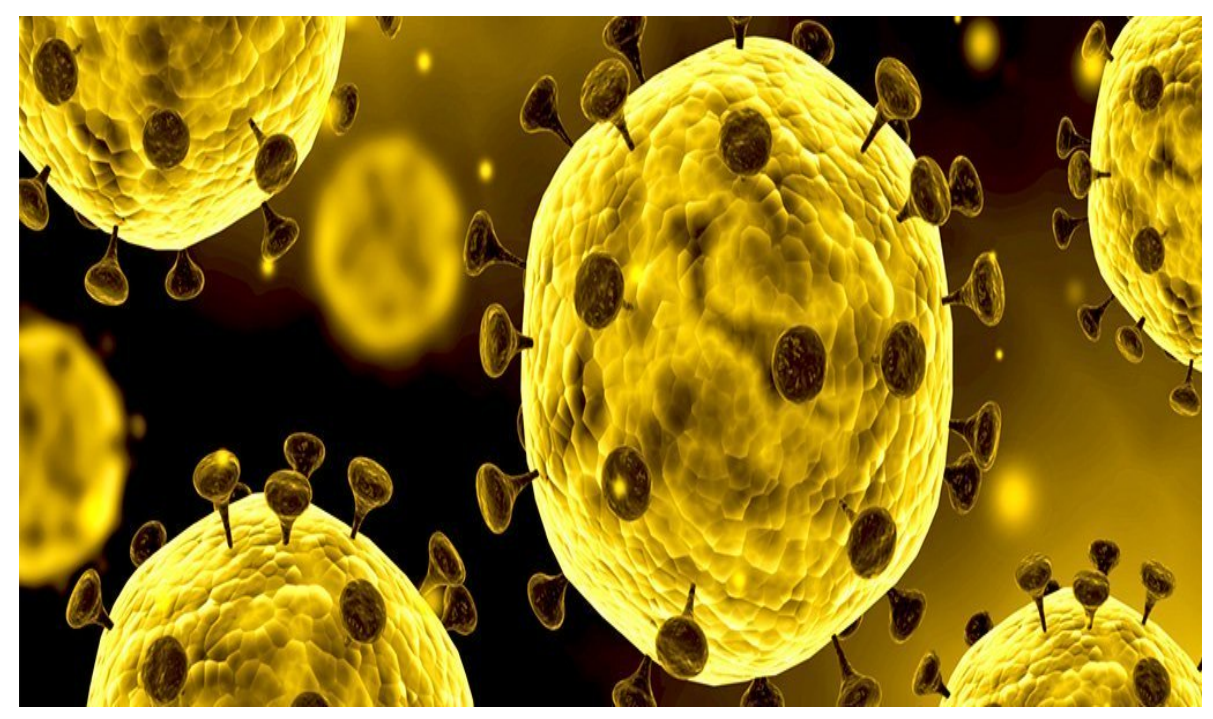

Fig. 1. An illustration of a coronavirus. (Stocktrek Images/Getty Images) 
Table 1. Day-to-day data of COVID-19 cases from January 22 to February 21

\begin{tabular}{|ll|ll|ll|ll|}
\hline Date & Cases & Date & Cases & Date & Cases & Date & Cases \\
\hline 22 Jan & 580 & 30 Jan & 9,823 & 07 Feb & 34,876 & 15 Feb & 69,197 \\
23 Jan & 845 & 31 Jan & 11,950 & 08 Feb & 37,552 & 16 Feb & 71,329 \\
24 Jan & 1,317 & 01 Feb & 14,553 & 09 Feb & 40,553 & 17 Feb & 73,332 \\
25 Jan & 2,015 & 02 Feb & 17,391 & 10 Feb & 43,099 & 18 Feb & 75,184 \\
26 Jan & 2,800 & 03 Feb & 20,630 & 11 Feb & 45,134 & 19 Feb & 75,700 \\
27 Jan & 4,581 & 04 Feb & 24,545 & 12 Feb & 59,287 & 20 Feb & 76,676 \\
28 Jan & 6,058 & 05 Feb & 28,266 & 13 Feb & 64,438 & 21 Feb & 77,673 \\
29 Jan & 7,813 & 06 Feb & 31,439 & 14 Feb & 67,100 & 22 Feb & 78,773 \\
\hline
\end{tabular}



Fig. 2. Day-to-day worldwide growth of COVID-19 cases from January 22 to February 21

Most of the researches found that the COVID-19 Coronavirus has come from a seafood market in Wuhan where more than 50 wild animals were sold illegally including endangered pangolins and now spreading from human to human through regular basis human contact- most cases by air. The first group of patients who got admitted to the hospital after affecting by the virus was workers and the customers of the market. After analysis and comparing the genetic sequences of COVID-19 with other known coronavirus, researchers have suggested that the new virus is most closely related to two bat SARS-like coronavirus samples from China and the virus has been transformed from snake to human as snake often hunts for bats. The scariest thing about the virus are that there is no perfect vaccine or specific treatment for this infectious disease, not even against SARS and MARS. Though research is ongoing to develop a new vaccine for this, it is a long process. These are really concerning issues similar to the previously spreading and life intimidation virus Zika and Ebola [4, 5]. 
Numerous models have been introduced To model the growth of biological systems. These variously addressed population dynamics, whether modelled discretely or continuously can predict the population dynamics with realistic intrinsic growth rate. Others model actual physical growth of some property of interest for an organism or organisms.

In this manuscript, our main purpose is to analyze the data for the recent outbreak of COVID-19 coronavirus and find the growth model that fits the data more perfectly. We hope that, this manuscript will be able to certify the importance of mathematical modeling to predict population dynamics.

\section{MALTHUSIAN MODEL}

To consider a single species continuous growth model of population $P$, initially a size of $P_{0}$ with per capita number of recruited individuals $b$ and recovered individuals $d$ in disease outbreak dynamics (basically birth and death respectively) can be demonstrated through [9]

$$
P^{\prime}(t)=(b-d) P(t), \text { for } t \in(0, \infty),
$$

where $(b-d)$ can be noted as per capita growth rate or propagation rate of the disease $r$. So

$$
P^{\prime}(t)=r P(t), \text { for } t \in(0, \infty),
$$

with the model solution

$$
P(t)=P_{0} e^{r t}, \text { for } t \in(0, \infty) .
$$

Here $r$ is the intrinsic growth rate and so represents growth rate per capita with the biological interpretation that $r>0$ means the outbreak of the infection and $r<0$ means that the infection is shutting down spreading. This solution is not much realistic as no population can grow boundlessly because of limited food supply, decreasing limited space reservedness for individuals et-cetera. But this simple exponential growth model can provide an adequate approximation to such growth for the initial period.

\section{LOGISTIC GROWTH MODEL}

In 1838 P.F. Verhulst [10] considered after a certain population size the environment will pull up the growth rate to make a significant balance in nature.

$$
P^{\prime}(t)=r P(t)\left(1-\frac{P(t)}{K}\right), \text { for } t \in(0, \infty),
$$

where, $r$ and $K$ are positive constants. Here $K$ is a self-limiting process called "carrying capacity" or "saturation level" which depends on the available resources in nature. Verhulst named this growth as logistic growth in a population.

Lotkas analysis [11] of this logistic growth showed that $\frac{\mathrm{d} P}{\mathrm{~d} t}$, the rate of population growth at any time $t$ is a function of the population size at that time, $P(t)$, that is, for any $t \in(0, \infty)$,

$$
P^{\prime}(t)=f(P(t))
$$

At zero population $f(0)=0$, has zero growth $P^{\prime}=0$, so $P=0$ must be an algebraic root of the yet unknown function $f(P)$. Hence Taylor series expansion near $P=0$ yields the following power series

$$
\begin{aligned}
f(P) & =P f^{\prime}(0)+\frac{P^{2}}{2 !} f^{\prime \prime}(0) \\
& =P\left[f^{\prime}(0)+\frac{P}{2} f^{\prime \prime}(0)\right]
\end{aligned}
$$

where, Lotka [11] assumed the higher terms are negligible.

Now, by setting $f^{\prime}(0)=r$ and $f^{\prime \prime}(0)=$ $-\frac{2 r}{K}$, where $r$ is the intrinsic growth rate of the population and $K$ is the carrying capacity, Lotkas analysis heads to the Verhulst logistic equation (3.1).

In the Verhulst-Pearl equation, the Verhulst logistic equation (3.1) was also referred to in the literature after Verhulst. They first derived the curve, and Pearl [12] used the curve to approximate population growth in the United States in 1920. 
The solution of the Verhulst logistic model (3.1) is $P(t)=\frac{K P_{0}}{P_{0}+\left(K-P_{0}\right) e^{-r t}}$, for $t \in(0, \infty)$,

where $P(0)=P_{0}$.

Calculating the solution (3.2) we observe

- when $P_{0}<K, P^{\prime}>0, P(t) \rightarrow K$ as $t \rightarrow \infty$.

- when $P_{0}=K, P^{\prime}=0, P(t) \rightarrow K$ as $t \rightarrow \infty$.

- when $P_{0}>K, P^{\prime}<0, P(t) \rightarrow K$ as $t \rightarrow \infty$.

- for each situation $P(t) \rightarrow K$ as $t \rightarrow \infty$, so the population will ultimately reach its carrying capacity anyhow.

- the relative growth rate, $\frac{P^{\prime}}{P}$, declines linearly with increasing population size.

- at the point of inflection the population is exactly equals to the half of the carrying capacity, i.e. $P_{\mathrm{inf}}=\frac{K}{2}$.

When $r>0$, the resulting growth curve has a sigmoidal shape and, from (3.2), is asymptotic to the carrying capacity $K$. For $r<0$ and a reduction in the growth rate per capita is present, the growth curve is asymptotic to zero leading to population extinction. In the trivial case of no intrinsic growth rate, $r=0$, the population remains static at the initial value of $P_{0}$. Population biologists and ecologists are interested mainly in the case where $r>0$ and we restrict our study to this case in this paper.

\section{NUMERICAL COMPARISON}

We took the real situation data from a very well trusted link the ALA (American Library Association) certified worldometer [8], where they update the daily data with authenticity about different global issues and the data is presented here in the Table 1.

Now, here the comparison is done with numerical presentations in Figs. 3-6.

From these Fig. 3 it is clear that, the exponential growth is almost similar to the real data for a very short initial time. But the logistic growth prediction is almost reflects the real graph.

Fig. 4 shows that, the growth of COVID-19 outside of China, the heart of the outbreak, is quite increasing like as an exponential growth. And considering the initiatives taken all over the world as logistic constant, logistic growth prediction is a better simulation in this case.

The active cases shown in Fig. 5 indicates a carrying capacity from which peak point the case number started going down. So, it follows from the study that, logictic growth prediction can give a much more good prediction than the Malthusian prediction can do.
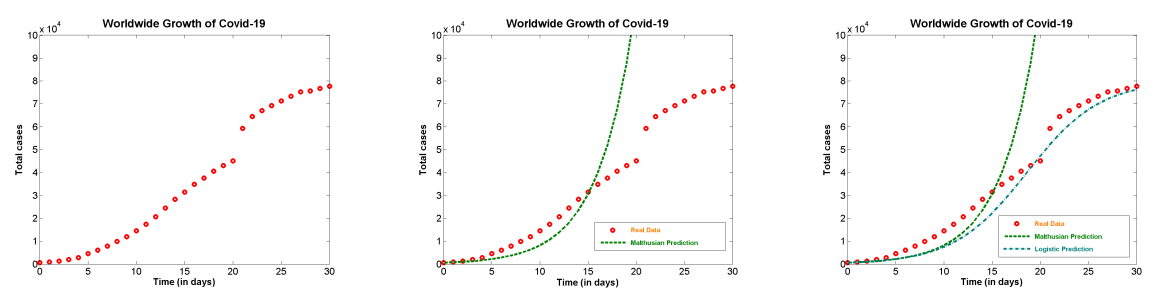

Fig. 3. Real life data versus mathematical predictions

In our last comparison, we present the graphical presentation of the total number of cured population that are recovered and discharged from hospital from February 02 to February 23, 2020. Our observation concludes here with a proper prediction of logistic growth function than a slight infraction for the Malthusian prediction. 

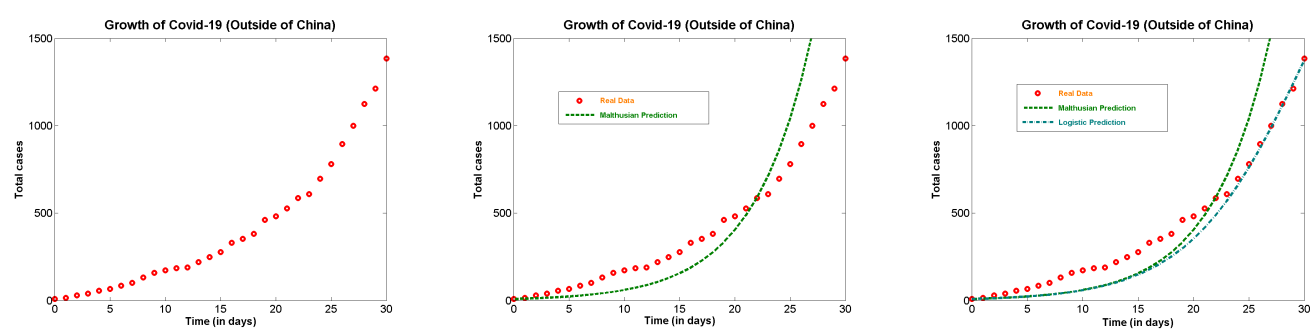

Fig. 4. Real life data versus mathematical predictions
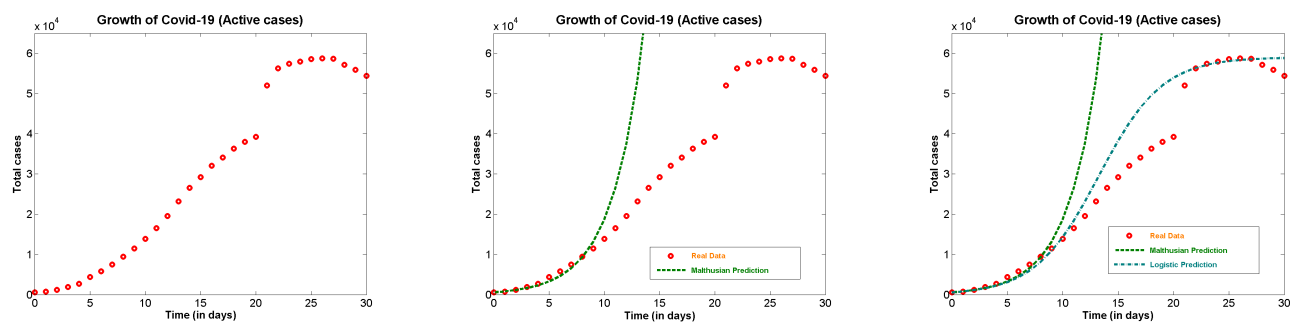

Fig. 5. Real life data versus mathematical predictions
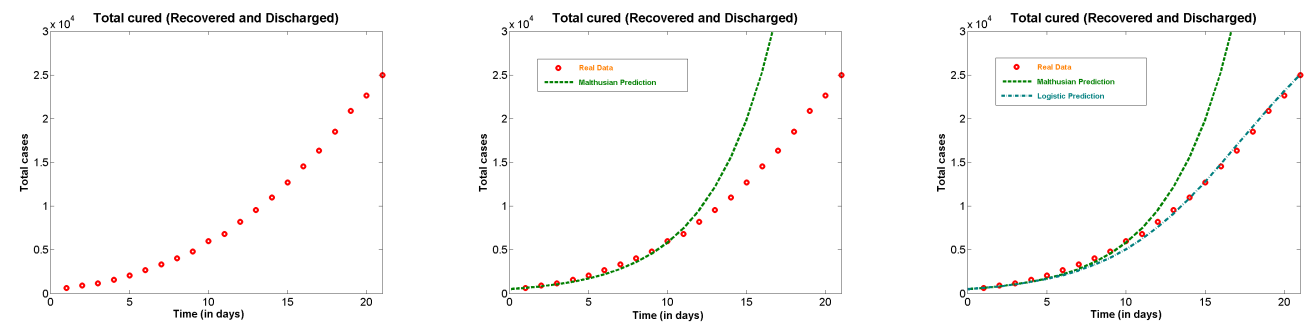

Fig. 6. Real life data versus mathematical predictions

Note: All data has been taken from worldometer [8] on February $24^{\text {th }}, 2020$.

\section{CONCLUSION}

Mathematical and computational models are increasingly used to help interpret biomedical data produced by high-throughput genomics and proteomics projects. The application of advanced computer models enabling the simulation of complex biological processes generates hypotheses and suggests experiments. Appropriately interfaced with biomedical databases, models are necessary for rapid access to, and sharing of knowledge through data mining and knowledge discovery approaches.

In this context, this manuscript presents two basic and simple growth function often used to modify for specific dynamical population issues. We graphically analyzed the data and compared with the Malthusian exponential growth prediction with the corresponding intrinsic growth rates and then with the Verhulst [10] logistic model with 
both the corresponding intrinsic growth rates and cappying capacity that knows how to and how much to pull-up the rein to have a realistic outcome.

\section{ACKNOWLEDGEMENT}

The authors acknowledged to the anonymous reviewers for their constructive suggestions to revise the manuscript. The first author $M$. Kamrujjaman research was partially supported by TWAS grant: 2019_19-169 RG/MATHS/AS_I.

\section{COMPETING INTERESTS}

Authors have declared that no competing interests exist.

\section{REFERENCES}

[1] Ahmad T, Khan M, Haroon TH, Musa SN, Hui J, Bonilla-Aldana DK, RodriguezMorales AJ, COVID-19: Zoonotic aspects. Travel Medicine and Infectious Disease, ELSEVIER; 2020.

[2] Zhao S, Musa SS, Lin Q, . . \& Wang MH. Estimating the unreported number of Novel Coronavirus (2019-nCoV) cases in China in the first half of january 2020: A data-driven modelling analysis of the early outbreak. J. Clin. Med. 2020;9(2):388.

[3] Chen N, Zhou M, Dong X, ... \& Zhang L. Epidemiological and clinical characteristics of 99 cases of 2019 Novel Coronavirus pneumonia in Wuhan, China: A descriptive study. The Lancet. 2020;395(10223):507513.

[4] Kamrujjaman M, Hossain MA. Spreading reason of 2019-nCoV and the message of Almighty God (Allah): A justification for the protection of mankind. Arabian Journal of Medical Sciences. 2020;3(1):8-10.

[5] World Health Organization (WHO); 2020.

[6] Lu R, Zhao X, Li X, ... \& Tan W. Genomic characterisation and epidemiology of 2019 novel coronavirus: Implications for virus origins and receptor binding. The Lancet. 2020;395(10224):565-574.

[7] Ji W, Wang W, Zhao X, Zai J, Li X. Homologous recombination within the spike glycoprotein of the newly identified coronavirus may boost crossspecies transmission from snake to human. Journal of Medical Virology. 2020;92(4):1-29.

[8] 'worldometer', COVID-19 CORONAVIRUS / CASES; 2020.

[9] Malthus T. An essay on the principle of population. London; 1798.

[10] Verhulst PF. Notice sur la loi que la population suit dans son accroissement. Corr. Math. Physics. 1838;10:113.

[11] Lotka AJ. Elements of mathematical biology. DOVER, New York; 1956.

[12] Pearl R, Reed LJ. On the rate of growth of the population of United States since 1790 and its mathematical representation. Proceedings National Academy of Sciences, USA. 1920;275.

(C) 2020 Kamrujjaman et al.; This is an Open Access article distributed under the terms of the Creative Commons Attribution License (http://creativecommons.org/licenses/by/4.0), which permits unrestricted use, distribution, and reproduction in any medium, provided the original work is properly cited. 